03.3

\title{
Динамика излучения наносекундного поверхностного скользящего разряда в потоке с ударной волной
}

\author{
(ㄱ А.Ю. Кузнецов, И.В. Мурсенкова, П.Ю. Уланов \\ Московский государственный университет им. М.В. Ломоносова, Москва, Россия \\ E-mail: kuznecov.alexander15@gmail.com
}

Поступило в Редакцию 13 июня 2019г.

В окончательной редакции 25 июня 2019г.

Принято к публикации 23 сентября 2019 г.

\begin{abstract}
Экспериментально исследовано пространственно-временно́е распределение излучения поверхностного скользящего разряда длительностью $\sim 300 \mathrm{~ns}$ в неподвижном воздухе при давлениях 2-200 Torr и при взаимодействии с плоскими ударными волнами с числами Маха 2.8-3.3. Проанализированы динамика излучения разряда на основе обработки разверток и 9-кадровых изображений свечения, спектральный состав излучения и ток разряда. Показано, что зависимость интенсивности излучения разряда, взаимодействующего с ударной волной, коррелирует с моделируемой временно́й зависимостью заселенности состояния $C^{3} \Pi_{u}$ азота при ударном сжатии плазменной области.
\end{abstract}

Ключевые слова: поверхностный скользящий разряд, динамика излучения, время затухания, электроннооптическая камера, плоская ударная волна.

DOI: 10.21883/PJTF.2019.24.48804.17924

Изучение взаимодействия потоков газа с плазменными образованиями связано с задачами плазменной аэродинамики и остается актуальным на протяжении последних десятилетий [1-3]. При помощи поверхностных разрядов разного типа (плазменных актуаторов) можно реализовать энерговклад в пристеночную область течения газа, контролируя ламинарно-турбулентный переход в пограничном слое, положение зон отрыва и скачков уплотнения вблизи обтекаемой поверхности [1]. Поверхностный скользящий разряд длительностью $\sim 300 \mathrm{~ns}$ оказывает ударно-волновое и тепловое воздействие на однородные высокоскоростные потоки [3]. В присутствии фронта ударной волны изменяется пространственная структура свечения наносекундных разрядов, и воздействие на поток определяется последующим движением газодинамических разрывов [4]. Понимание механизма взаимодействия ударных волн с газоразрядной плазмой, включая определение динамики излучения разряда вблизи фронта ударной волны, дает возможность определять оптимальные режимы применения разрядов для эффективного управления неоднородными потоками с разрывами. Целью настоящей работы является экспериментальное исследование пространственно-временно́го распределения свечения, спектрального состава излучения и тока наносекундного поверхностного скользящего разряда в неподвижном воздухе при давлении 2-200 Torr (плотность $0.003-0.330 \mathrm{~kg} / \mathrm{m}^{3}$ ) и в сверхзвуковых потоках воздуха с плоскими ударными волнами с числами Маха $2.8-3.3$.

Экспериментальные исследования проводились на ударной трубе с прямоугольным каналом сечением $24 \times 48 \mathrm{~mm}$ и встроенной разрядной камерой $[3,4]$. На верхней и нижней стенках разрядной камеры на рас- стоянии $24 \mathrm{~mm}$ друг от друга инициировались поверхностные скользящие распределенные разряды протяженностью $10 \mathrm{~cm}$ (по направлению движения ударной волны) с межэлектродным расстоянием $3 \mathrm{~cm}$. Скользящий разряд формировался в тонком газовом слое вблизи поверхности диэлектрика (стеклотекстолита) при подаче импульсного напряжения $25 \mathrm{kV}$ на систему электродов специальной конфигурации [3]. Исследовалось излучение разрядов в неподвижном воздухе и в сверхзвуковых потоках с плоской ударной волной при различных положениях фронта ударной волны в разрядной области и за ее пределами. За время тока разряда фронт ударной волны смещался менее чем на $0.5 \mathrm{~mm}$. С помощью сигналов пьезоэлектрических датчиков давления в канале ударной трубы измерялась скорость ударных волн и осуществлялась синхронизация процессов.

Через боковые стенки разрядной камеры, выполненные из кварцевого стекла с полосой пропускания 200-2800 nm, фотокамерами и высокоскоростными электронно-оптическими камерами БИФО К008, К011 регистрировалось свечение разряда, спектрометром AvaSpec-2048FT (диапазон длин волн 200-1100 nm) спектры излучения. Длительность разверток свечения, полученных с помощью электронно-оптической камеры БИФО К008, составляла $2 \mu \mathrm{s}$. Щель камеры располагалась вдоль оси $O X$, поперек направления распространения каналов разряда и вдоль направления потока (рис. 1). При 9-кадровой регистрации камерой БИФО К011 экспозиция составляла $100 \mathrm{~ns}$, пауза между кадрами 100 или $200 \mathrm{~ns}$. Диапазон спектральной чувствительности электронно-оптических камер 270-850 nm (К008), 370-850 nm (К011). Регистрирующая излучение аппаратура устанавливалась под углом к плоскости раз- 
$a$
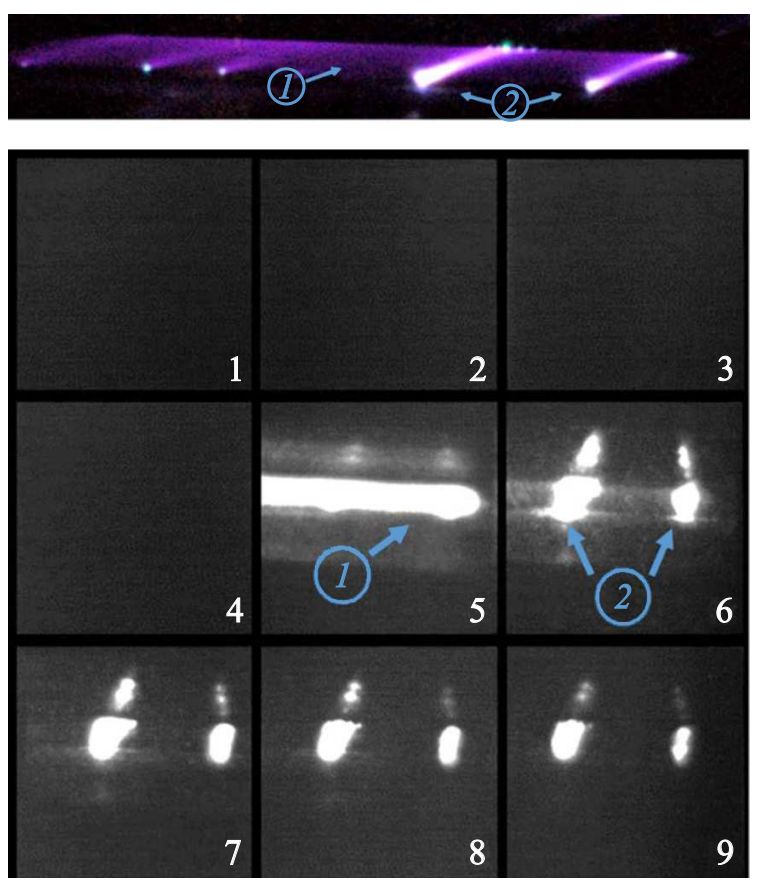
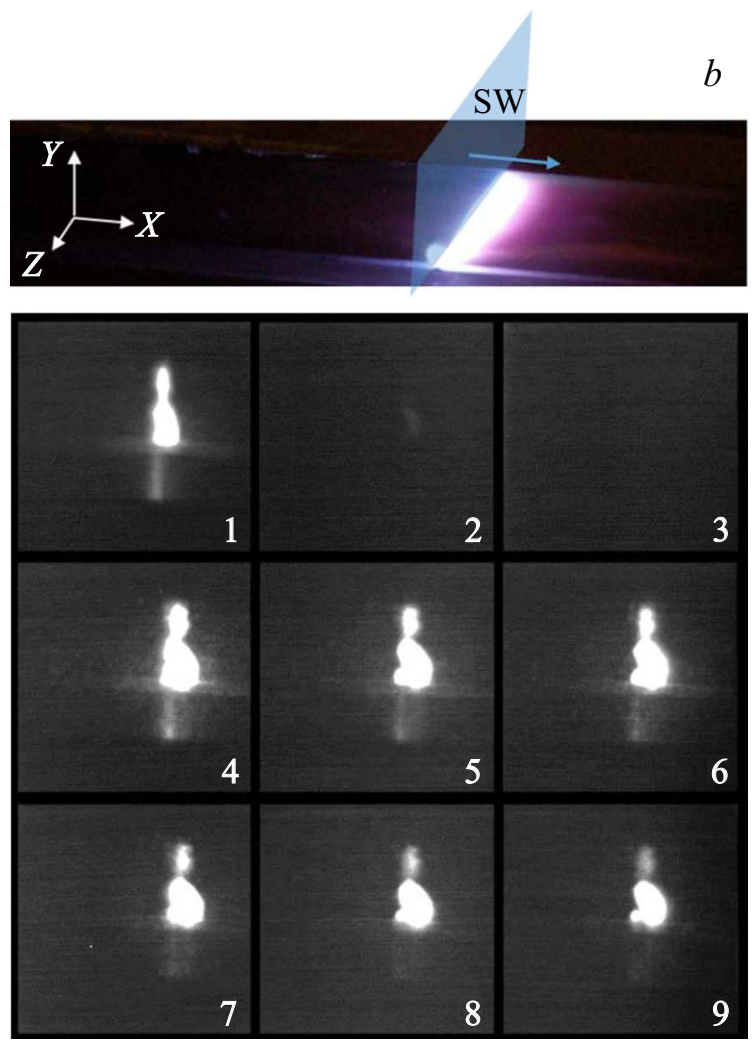

Рис. 1. Фотоизображения (вверху) и 9-кадровые изображения (внизу) свечения поверхностного скользящего разряда в неподвижном воздухе при давлении 32 Torr $(a)$ и при взаимодействии с фронтом ударной волны $(b)$. Направление движения ударной волны показано стрелкой, число Маха 3.0, давление перед фронтом 17 Torr, фронт ударной волны за пределами разрядного промежутка (на расстоянии $2 \mathrm{~mm}$ ). На 9-кадровых изображениях экспозиция кадра $100 \mathrm{~ns}$, пауза между кадрами $100(a)$ и $200 \mathrm{~ns}(b)$.

ряда в конце разрядного промежутка (по направлению движения ударной волны). По разверткам свечения определялись зависимости интенсивности свечения разрядов от времени и времена затухания свечения при разных условиях инициирования разрядов, по 9-кадровым изображениям анализировалось пространственное распределение свечения. С помощью специального малоиндуктивного шунта измерялся ток разряда.

Спектральные исследования показали, что основной вклад в излучение поверхностного скользящего разряда вносит вторая положительная система азота, соответствующая переходам $C^{3} \Pi_{u} \rightarrow B^{3} \Pi_{g} \quad(281-498 \mathrm{~nm})$. В спектрах наблюдаются линии атомов азота, кислорода и водорода $\mathrm{H}_{\alpha}$ и $\mathrm{H}_{\beta}$. Линии водорода присутствуют вследствие диссоциации молекул воды, содержащихся в воздухе. Концентрация электронов в плазме в неподвижном воздухе, рассчитанная по штарковскому уширению линий водорода, при экспериментальных условиях составляла $(0.1-1.4) \cdot 10^{15} \mathrm{~cm}^{-3}$. После рекомбинации в течение 100-1000 ns в области неравновесного возбужденного газа происходила более медленная релаксация электронного и колебательного возбуждения молекул [5].

На рис. 1, а приведено фотоизображение разряда в неподвижном воздухе, на котором наблюдается диффузное свечение плазменного слоя, состоящего из каналов близкой интенсивности $(1)$, и интенсивное свечение ярких каналов (2). На соответствующем 9-кадровом изображении на рис. $1, a$ на кадре 5 видно свечение диффузного слоя (1) и двух ярких каналов (2), на следующих кадрах наблюдается затухающее излучение каналов. Временны́е зависимости интенсивности свечения каналов разряда в неподвижном воздухе (рис. 2, a) показывают, что свечение яркого канала имеет значительно бо́льшую длительность и коррелирует с осцилляциями тока (рис. 2, b). Полная длительность диффузного свечения меньше $200 \mathrm{~ns}$, длительность свечения ярких каналов могла превышать $2000 \mathrm{~ns}$. На рис. 3 показаны времена затухания излучения каналов разряда в неподвижном воздухе при разных давлениях, определенные по разверткам свечения. Время затухания диффузного излучения при давлении до 60 Torr близко к времени жизни излучающего состояния азота $C^{3} \Pi_{u}(\sim 40 \mathrm{~ns})$, затем с ростом давления оно увеличивается до $100 \mathrm{~ns}$. Времена затухания излучения ярких каналов разной интенсивности выше и лежат в пределах 100-350 ns. Динамика излучения интенсивных каналов разряда может быть связана с заселением состояний $C^{3} \Pi_{u}$ и $B^{3} \Pi_{g}$, которые образуются при столкновениях молекул азота 
в метастабильном состоянии $A^{3} \Sigma_{u}^{+}[6-8]$ в следующих реакциях [9]:

$$
\begin{aligned}
\mathrm{N}_{2}\left(A^{3} \Sigma_{u}^{+}\right) & +\mathrm{N}_{2}\left(A^{3} \Sigma_{u}^{+}\right) \rightarrow \mathrm{N}_{2}\left(C^{3} \Pi_{u}, v_{c}=0-4\right) \\
& +\mathrm{N}_{2}\left(X^{1} \Sigma_{g}^{+}, v=0\right) \\
\mathrm{N}_{2}\left(A^{3} \Sigma_{u}^{+}\right) & +\mathrm{N}_{2}\left(B^{3} \Pi_{g}\right) \rightarrow \mathrm{N}_{2}\left(C^{3} \Pi_{u}, v_{c}=0-4\right) \\
& +\mathrm{N}_{2}\left(X^{1} \Sigma_{g}^{+}, v=0\right), \\
\mathrm{N}_{2}\left(A^{3} \Sigma_{u}^{+}\right)+ & \mathrm{N}_{2}\left(A^{3} \Sigma_{u}^{+}\right) \rightarrow \mathrm{N}_{2}\left(B^{3} \Pi_{g}\right),+\mathrm{N}_{2}\left(X^{1} \Sigma_{g}^{+}\right)
\end{aligned}
$$

Концентрация молекул азота в метастабильном состоянии $A^{3} \Sigma_{u}^{+}$в наносекундных разрядах может достигать значений $10^{14} \mathrm{~cm}^{-3}[6,7]$.

При инициировании разряда в присутствии плоской ударной волны внутри разрядного промежутка ток протекает перед фронтом волны, в области низкой плотности, как показали фотоизображения и 9-кадровые изображения свечения, а также теневые исследования поля течения после разряда [10]. В том случае, когда ударная волна находится на небольшом расстоянии за пределами разрядного промежутка, ток разряда протекает по П-образным каналам, включающим фронт исходной ударной волны (рис. $1, b$ ). Спектр излучения разряда при этом содержит континуум и интенсивные линии атомов азота, кислорода, водорода. Концентрация электронов в плазменной области вблизи фронта ударной
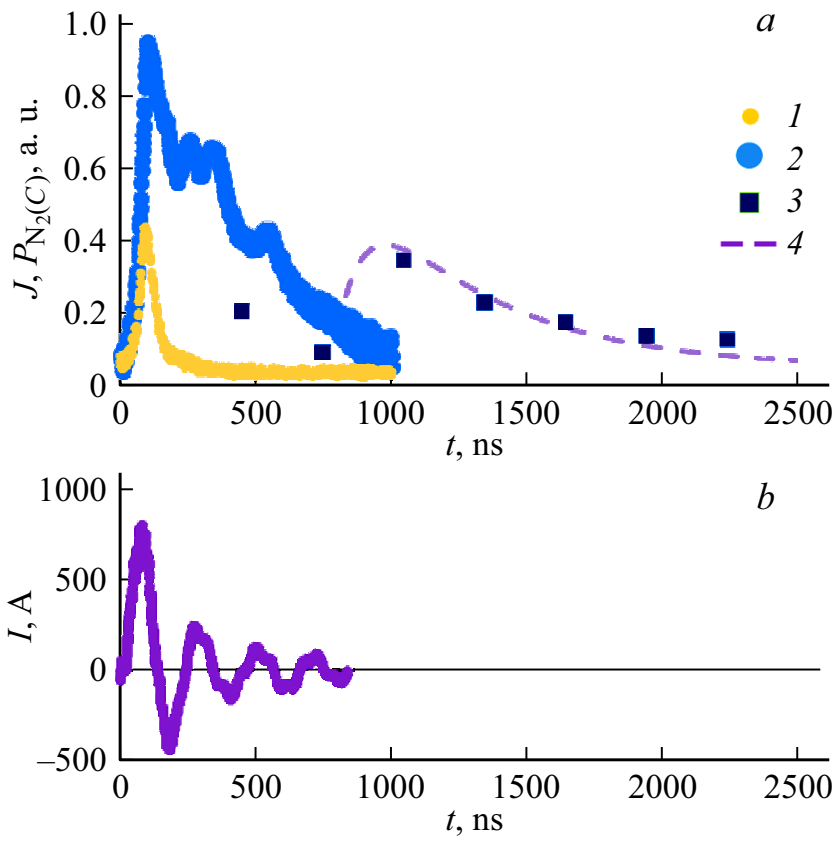

Pис. 2. $a-$ временны́е зависимости интенсивности свечения $J$ диффузного (1) и яркого (2) каналов разряда в неподвижном воздухе при давлении 25 Torr, интенсивности свечения разряда, взаимодействующего с фронтом ударной волны (3), заселенности $P_{\mathrm{N}_{2}(C)}$ состояния $\mathrm{N}_{2}\left(C^{3} \Pi_{u}\right)(4)$, расчет (зависимости 3 и 4 приведены для условий, соответствующих рис. $1, b) ; b-$ временна́я зависимость тока разряда в неподвижном воздухе при давлении 25 Torr.

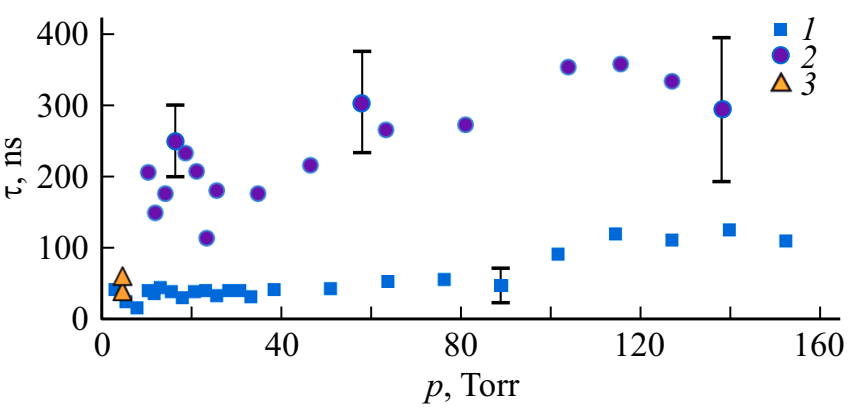

Рис. 3. Времена затухания свечения диффузных (1) и интенсивных (2) каналов поверхностного скользящего разряда в зависимости от давления воздуха, а также время спада заселенности состояния $\mathrm{N}_{2}\left(C^{3} \Pi_{u}\right)$ в ближнем послесвечении наносекундного объемного разряда при давлении воздуха 4.6 Torr (3) [6].

волны на порядок выше, чем в неподвижном воздухе. Длительность излучения разряда, взаимодействующего с фронтом ударной волны, значительно больше длительности свечения разряда в неподвижном воздухе (рис. 2, a, кривая 3) и зависит от исходного положения плоской ударной волны в разрядном промежутке. Динамика излучения характеризуется тем, что после прекращения тока разряда наблюдается нарастание интенсивности излучения в течение 200-300 ns, а затем постепенное затухание (рис. 2, a, символы 3). Время затухания излучения разряда, взаимодействующего с ударной волной, определяется числом Маха ударной волны и начальным положением фронта и составляет 600-2500 ns. Подобные особенности излучения наблюдались при взаимодействии импульсного объемного разряда с плоской ударной волной [8].

В случае взаимодействия пристеночной плазменной области с плоской ударной волной динамику свечения нужно рассматривать с учетом движения газодинамических разрывов, образовавшихся после инициирования разряда. При импульсном энерговкладе происходят распады газодинамических разрывов на границах плазма-воздух. В результате распада разрыва на фонте исходной ударной волны образуются контактная поверхность и две ударные волны [4,8], одна из которых движется по области рекомбинирующей и релаксирующей плазмы, сжимая ее и создавая зону с повышенной концентрацией молекул азота в возбужденных состояниях.

Было проведено моделирование зависимости заселенности состояния $\mathrm{N}_{2}\left(C^{3} \Pi_{u}\right)$ от времени при движении плоской ударной волны по плазменной области в соответствии с реакциями (1)-(3) после прекращения тока разряда. В качестве начального условия в области движения ударной волны задавалась временна́я зависимость концентрации молекул азота в состоянии $A^{3} \Sigma_{u}^{+}$из работы [6]. При повышенной после прохождения ударной волны концентрации молекул азота в этом состоянии повышается заселенность излучающего состояния $C^{3} \Pi_{u}$, что приводит к продолжительному послесвечению узкой 
области вблизи фронта ударной волны после разряда. Полученная модельная зависимость (рис. 2, $a$, кривая 4) хорошо коррелирует с экспериментальной зависимостью интенсивности излучения разряда в присутствии плоской ударной волны (рис. 2, а, символы 3). Таким образом, наблюдаемая динамика излучения разряда, взаимодействующего с фронтом ударной волны, определяется изменением заселенности состояния $C^{3} \Pi_{u}$ молекулярного азота при ударном сжатии области релаксирующей плазмы в условиях проведенных экспериментов.

Проведенное исследование показало, что геометрия протекания тока наносекундного поверхностного скользящего разряда в воздухе, временнб́е характеристики излучения и соответственно динамика энерговклада зависят от характера взаимодействия разряда с фронтом плоской ударной волны. При числах Маха ударных волн $2.8-3.3$ в зависимости от положения фронта в момент инициирования разряда длительность послесвечения может превышать $2000 \mathrm{~ns}$, а время затухания излучения достигает $2500 \mathrm{~ns}$, что значительно больше времени затухания разряда в неподвижном воздухе. Временна́я зависимость излучения вблизи фронта ударной волны после разряда может быть связана с повышением концентрации молекул азота в метастабильном состоянии $A^{3} \Sigma_{u}^{+}$при ударном сжатии области релаксирующей плазмы. Результаты исследования следует учитывать при разработке плазменных устройств на основе разрядов наносекундной длительности, предназначенных для управления высокоскоростными потоками газа с ударными волнами.

\section{Финансирование работы}

Работа выполнена при финансовой поддержке Российского фонда фундаментальных исследований (грант 1908-00661) и Программы развития МГУ до 2020 г.

\section{Конфликт интересов}

Авторы заявляют, что у них нет конфликта интересов.

\section{Список литературы}

[1] Leonov S.V., Adamovich I.V., Soloviev V.R. // Plasma Sources Sci. Technol. 2016. V. 25. P. 063001 (1-32). DOI: https://doi.org/10.1088/0963-0252/25/6/063001

[2] Голуб В.В., Савельев А.С., Сеченов В.А., Сон Э.Е., Терешонок Д.В. // ТВТ. 2010. Т. 48. № 6. С. 948-955.

[3] Mursenkova I.V., Znamenskaya I.A., Lutsky A.E. // J. Phys. D: Appl. Phys. 2018. V. 51. P. 105201.

DOI: https://doi.org/10.1088/1361-6463/aaa838

[4] Дорощенко И.А., Знаменская И.А., Кузнеиов А.Ю., Мурсенкова И.В., Сысоев Н.Н. // ЖТФ. 2018. Т. 88. В. 5. С. 684 691.

[5] Райзер Ю.П. Физика газового разряда. М.: Наука, 1987. $592 \mathrm{c}$.
[6] Mintoussov E.I., Pendleton S.J., Gerbault F.G., Popov N.A., Starikovskaia S.M. // J. Phys. D: Appl. Phys. 2011. V. 44. P. 285202 (1-13). DOI: 10.1088/0022-3727/44/28/285202

[7] Nagaraja S., Yang V., Adamovich I. // J. Phys. D: Appl. Phys. 2013. V. 46. P. 155205 (1-13).

DOI: https://doi.org/10.1088/0022-3727/46/15/155205

[8] Кузнещов А.Ю., Мурсенкова И.В. // Прикладная физика. 2016. № 5. C. 16-21.

[9] Лебедев Ю.А., Шахатов В.А. // Физика плазмы. 2006. T. 32. № 1. C. 58-74.

[10] Мурсенкова И.В., Кузнецов А.Ю., Сазонов А.С. Экспериментальное исследование влияния импульсных скользящих разрядов на течение с ударной волной в канале // Материалы XII Междунар. конф. по прикладной математике и механике в аэрокосмической отрасли (NPNJ'2018). М.: МАИ, 2018. С. 278-280. 